\title{
Contrast- and Illumination-Invariant Object Recognition from Active Sensation
}

\author{
Ingo Rentschler ${ }^{1}$, Erol Osman ${ }^{1} \&$ Martin Jüttner ${ }^{2}$
}

\section{Reference for citations:}

Rentschler, I., Osman, E. \& Jüttner (2009). Contrast-invariant object recognition from active sensation. Spatial Vision 22, 383-396.

\footnotetext{
${ }^{1}$ Institute of Medical Psychology, University of Munich, Munich, Germany

${ }^{2}$ Psychology, School of Life and Health Sciences, Aston University, Birmingham, UK
}

\begin{abstract}
It has been suggested that the deleterious effect of contrast reversal on visual recognition is unique to faces, not objects. Here we show from priming, supervised category learning, and generalization that there is no such thing as general invariance of recognition of non-face objects against contrast reversal and, likewise, changes in direction of illumination. However, when recognition varies with rendering conditions, invariance may be restored, and effects of continuous learning may be reduced, by providing prior object knowledge from active sensation. Our findings suggest that the degree of contrast invariance achieved reflects functional characteristics of object representations learned in a task-dependent fashion.
\end{abstract}

Key words: Object recognition, invariant recognition, contrast reversal, direction of illumination, categorisation 


\section{Introduction}

Contrast reversal disrupts the visual recognition of human faces from grey-level (Galper, 1970; Hayes, Morrone, \& Burr, 1986; Liu, Collin, Burton \& Chaudhuri, 1999) and two-tone images (Phillips, 1972), whereas little or no effect of contrast reversal on the recognition of chairs (Subramaniam \& Biederman, 1997) and contraptions of geometric components (“Greebles”; Vuong, Peissig, Harrison \& Tarr, 2005) has been reported. A possible explanation of this discrepancy is the existence in test objects of differences in contrast-invariant shape features resulting from discontinuities of orientation and depth (Nederhouser, Yue, Mangini, \& Biederman, 2007). To exclude this possibility, the latter authors compared the effects of contrast reversal on the recognition of faces and non-face stimuli composed of spherical harmonics. The results obtained were suggestive to these authors of contrast reversal being deleterious to the recognition of faces but not objects.

An alternative account of the dependence of visual recognition on contrast reversal has been offered by Jüttner, Langguth \& Rentschler (2004). These authors studied supervised category learning (Caelli, Rentschler \& Scheidler, 1987) and generalisation to contrast reversal for several sets of compound Gabor signals (greylevel patterns composed of Gabor waveforms; Caelli, Hübner, \& Rentschler, 1986). The sets differed either locally in their grouping in feature space or globally in the grey-level distribution of learning patterns. Jüttner and co-workers found an inverse relationship between the set-specific duration of category learning to criterion and generalisation to contrast reversal whereby fast learning coincided with invariance to contrast reversal. They analysed their behavioural data by means of computer simulations employing an evidence-based, structural pattern classifier (cf. Jain \& Hoffman, 1988; Caelli \& Dreier, 
1994; Jüttner, Caelli \& Rentschler, 1997). For a given categorisation task, Jüttner et al. obtained sets of part and part-relational attributes, or feature states, as solutions within this evidence-based system (EBS). They found the psychophysically observed speed of category learning to be directly proportional to the number of EBS solutions in search space. Importantly, fast learning was associated with high relative frequency of contrast-invariant feature states used for generating class descriptions.

On the one hand, the findings by Jüttner et al. (2004) indicate that invariance to contrast in pattern recognition depends on task- or context-dependent characteristics of learned representations (cf. Albright, 1995). On the other hand, the visual recognition of three-dimensional (3D) objects could involve more abstract types of representation in the brain (Biederman, 1987; Biederman and Kalocsai, 1997) that entail a generic, learning-independent invariance to contrast reversal. To resolve this issue, we examine the effects of changed rendering within a category learning paradigm involving unfamiliar 3D objects (cf. Osman, Pearce, Jüttner \& Rentschler, 2000; Rentschler, Gschwind, Brettel, Osman \& Caelli, 2008). More specifically, we study supervised category learning as well as generalisation to novel viewpoints and changed rendering using 3D objects composed of a fixed number of identical spheres. For probing the nature of object representations, we control the amount of prior knowledge subjects have about test objects. This is achieved through priming, a technique from memory research using the beneficial influence of pre-exposure to a stimulus in the absence of explicit instructions to remember the stimulus (see Biederman \& Cooper, 1991; Cooper, Schacter, Ballesteros, and Moore, 1992).

The conjecture of invariance characteristics of object recognition being taskdependent entails the possibility that internalised representations are susceptible to 
continuous learning (Tagscherer and Protzel, 1998; Tagscherer, 2001; Keith-Magee, 2001, unpublished Ph.D. dissertation, Curtin University of Technology, Perth, W.A.). That is, visual object recognition may not only be enabled by category learning but continuously modified by interfering input signals (cf. Edelman, 1987). We investigate this possibility in an additional experiment, where participants classify object views in standard rendering after having performed with test views in changed rendering.

\section{METHODS}

\section{Subjects}

45 subjects participated for pay. They ranged in age from 19 to 45 years and were free of ophthalmologic and neurological disorders.

\section{Stimuli}

Learning objects. A set of three test objects was constructed using four identical sphere parts in varying spatial arrangements (Fig. 1a). Objects were designed according to the concept of chirality. Chiral objects cannot be carried into their mirror images by rotations and translations. Handed chiral objects allow categorization into "left" and "right" objects (King, 2003). Bilaterally symmetric objects are achiral by definition. Thus, one non-handed achiral object (object 1, cf. Fig. 1a) and one pair of handed chiral objects (objects 2 and 3) were used. Physical models were constructed from directly connected polystyrene balls each measuring $6 \mathrm{~cm}$ in diameter. Three spheres formed a rectangular isosceles triangle. The fourth sphere was placed perpendicularly above the centre of one of the base spheres. Corresponding virtual models were generated and displayed for $250 \mathrm{~ms}$ each as perspective 2D projections by means of the Open 
Inventor $^{\mathrm{TM}}$ (Silicon Graphics, Inc.) 3D developer's toolkit. A lighting model of mixed directed and diffuse illumination lacking cast shadows was used. At the viewing distance of $1 \mathrm{~m}$, virtual objects subtended in average $7.4^{\circ}$.

Learning views. Discarding views redundant due to rotational symmetry, 22 learning views ( 6 for object 1 , and 8 for each of objects 2 and 3 ) were generated by sampling azimuth and polar angle of the viewing-sphere in $60^{\circ}$ steps. Steps were taken on three great circles inclined against each other by $60^{\circ}$. The equatorial plane was horizontal and contained the symmetry axis of each object (three-fold rotational symmetry of object 1 and two-fold rotational symmetries of objects 2 and 3). The origin of the coordinate system used for sampling was at the centre of gravity of the component spheres and thus on the symmetry axis of each object. To reduce occlusion artefacts, reference views (Fig. 1a) were chosen such that the objects' symmetry axes were perpendicular to the picture plane and no centre of any sphere was directly in the equatorial plane. An angle of rotation in the picture plane, randomly selected from the values of $0^{\circ}, 60^{\circ}, 120^{\circ}$, and $180^{\circ}$, was employed to prevent observers from using the frame of the computer screen as a reference. To examine the possibility of recognition depending on pixel-based similarities of input signals and stored 2D views (cf. Bülthoff \& Edelman, 1992), classification probabilities were predicted from a Minimum-Distance Classifier (see Ahmed \& Rao, 1975) using the maximum of the 2D cross-correlation function as a measure of similarity (cf. Caelli \& Rentschler, 1986). Predicted classification probabilities were at chance level for all three objects, demonstrating that no differences of pixel-based similarities exist within the learning set. Thus, the $2 \mathrm{D}$ views of the learning set qualify as "structure-only" stimuli.

[Figures 1 about here] 
Test views. In order to test spatial generalisation, 64 views from novel viewpoints were obtained from sampling the viewing sphere as above but in $30^{\circ}$ steps. Views redundant due to rotational symmetry or identical to those used during supervised learning were discarded. Finally, the views were manipulated using three different rendering conditions. For views in the standard rendering condition the lighting model remained unaltered. In the contrast reversal condition, the grey level of each pixel was subtracted from the maximum value 255 to obtain reversed intensities (Figs. 2a). For views in the condition of changed direction of illumination (Fig. 2b), the default position of the light source was changed from the upper left (infinite distance, $10^{\circ}$ to the left and up from the viewer's position) to the lower right from the observer's position (infinite distance, $45^{\circ}$ to the right and $90^{\circ}$ down from the viewer's position).

[Figure 2 about here]

\section{Procedure}

The main experiment consisted of three parts: priming, supervised category learning, and generalization. Subjects who had carried out the generalization test under the rendering conditions of contrast reversal or changed direction of illumination (see below) completed an additional generalisation re-test, using the test views of the standard rendering condition.

Priming phase. During priming subjects either explored with their hands the physical objects occluded from sight (active touch priming) or explored virtual models by grasping $2 \mathrm{D}$ views on the computer display with the mouse and a cursor and rotating them within the viewing sphere (active vision priming). Priming lasted for $5 \mathrm{~min}$ in either condition. No instruction other than the invitation to familiarize themselves with 
the objects was given. As a baseline for the two priming conditions a neutral (control) condition was included, where subjects entered the second part of the experiment, visual category learning, directly, i.e. without acquiring prior object knowledge. Participants were randomly assigned to one the three experimental conditions, resulting in 15 participants per condition.

Supervised category learning. The second part of the experiment involved a procedure of supervised learning partitioned into learning units (for details, see Rentschler, Jüttner \& Caelli, 1994; Osman et al., 2000). Each learning unit consisted of a learning phase and a test phase. During the learning phase each of the 22 views of the learning set was presented once for $250 \mathrm{~ms}$ and in random order, followed by the corresponding object label ("1", "2" or "3") displayed for 1 s. During the test phase, each learning view was presented once, whereupon the observer assigned it to one of the three objects. Upon completion of each test phase, participants received feedback as to the percent correct value of their responses. No instructions as to the possible duration of classification decisions were given. On average duration, a learning unit took 5 min to complete. The series of learning units terminated upon reaching a criterion of $90 \%$ correct classification.

Generalization test. The final part of the main experiment assessed generalization to novel viewpoints and to changes in rendering. Here, each of 64 novel test views of one rendering condition was shown three times for $250 \mathrm{~ms}$ and in random order. Upon each presentation, the observer had to assign the view to one of the object categories 1 to 3 . The 15 participants within each priming condition were randomly assigned to one of the three rendering conditions, resulting in 5 participants per priming and rendering condition. 
Generalization re-test. To test for effects of continuous learning, participants who had completed the generalisation test under the conditions of changed rendering (contrast reversal or changed direction of illumination) were tested again for generalisation to novel viewpoints, this time using the test views of the standard rendering condition. The same procedure as during the generalization test was employed. However, the test views were presented only once in random order.

\section{RESULTS}

Table 1 summarises durations of supervised category learning to criterion (90\% correct classification) for the control condition, where no prior knowledge about test objects was provided, as well as the two priming conditions of active vision and active touch. Learning duration, as measured by the number of learning units, is distinctly reduced by either form of priming relative to the control group.

\section{[Table 1 about here]}

From the confusion matrix of each observer, the percentage of correct scores for each of the three objects was computed to assess classification performance in the generalization test. Figures 3 a-c show mean generalization performance to novel viewpoints for the control condition and the priming conditions of active vision and active touch. Each graph shows three pairs of bars, with the left (bright) bar corresponding to generalization performance for non-handed object 1 and the right (dark) bar to mean performance for handed objects 2 and 3. In order to test the impact of priming and rendering, we analysed the data in terms of separate 3 (priming condition: control vs. active vision vs. active touch) x 3 (rendering condition: standard vs. contrast 
reversal vs. changed direction of illumination) ANOVAs for handed and non-handed objects. The analysis yielded a significant main effect of priming condition (non-handed object: $\mathrm{F}(2,36)=6.72, \mathrm{p}<0.01$; handed objects: $\mathrm{F}(2,36)=15.92, \mathrm{p}<0.001)$. Rendering condition significantly affected generalization in case of handed objects 2 and 3 $(\mathrm{F}(2,36)=6.02, \mathrm{p}<0.01)$ and marginally in case of non-handed object $1(\mathrm{~F}(2,36)=3.13$, $\mathrm{p}=0.05$ ). There was no significant interaction between the two factors (non-handed object: $\mathrm{F}(4,36)=1.80, \mathrm{p}=0.15$; handed objects: $\mathrm{F}(4,36)=0.55, \mathrm{p}=0.70)$.

[Figure 3 about here]

To further assess the effect of rendering, additional one-way ANOVAs were conducted for each priming condition and object type. For the control condition, there was a significant effect of rendering on generalisation for both object types (non-handed object: $\mathrm{F}(2,12)=7.36, \mathrm{p}<0.01$; handed objects: $\mathrm{F}(2,12)=16.69$, $\mathrm{p}<0.001)$. As illustrated in Fig. 3a and confirmed by post-hoc Bonferroni comparisons, contrast reversal significantly impeded generalisation for both the handed and non-handed object (all ps $<0.05)$. A change in the direction of illumination impeded generalization of the handed objects $(p<0.01)$ while leaving recognition of the non-handed object unaffected $(p$ $=0.99)$.

No significant effect of rendering on generalization was observed in case of active vision or active touch priming for both non-handed object 1 (active vision: $\mathrm{F}(2,12)=0.30, \mathrm{p}=0.75 ;$ active touch: $\mathrm{F}(2,12)=0.01, \mathrm{p}=0.99)$ and handed objects 2 and 3 (active vision: $\mathrm{F}(2,12)=0.64, \mathrm{p}=0.54 ;$ active touch: $\mathrm{F}(2,12)=1.37, \mathrm{p}=0.29$ ). For both types of objects, categorisation performance was invariant to contrast reversal and changes in the direction of illumination. Still, paired t-tests revealed that for priming from active vision performance for handed objects 2 and 3 was consistently lower than 
for non-handed object 1 in these two rendering conditions $(t(4)>3.26, p<0.05$ and $t(4)=7.36, p<0.01$, respectively), whereas there was no significant difference for the baseline condition of standard rendering $(\mathrm{t}(4)=1.98, \mathrm{p}=0.12)$. Priming from active touch yielded a generalisation performance for the two types of objects that differed significantly only in case of changed illumination $(\mathrm{t}(4)=4.55, \mathrm{p}<0.05)$ but not for standard rendering $(\mathrm{t}(4)=0.27, \mathrm{p}=0.80)$ or contrast reversal $(\mathrm{t}(4)=2.10, \mathrm{p}=0.11)$,

[Figure 4 about here]

Figure 4 a-c shows results obtained in the re-test of spatial generalization to standard rendering for those subjects who had first been tested in the conditions of contrast reversal or changed direction of illumination (middle and right double bars). For comparison, generalization performance of the not re-tested group of participants (i.e., those who had directly been tested with novel views under conditions of standard rendering) is re-plotted from Figure 3 (open bars).

Separate 3 (priming condition: control vs. active vision vs. active touch) x 3 (rendering condition: standard direct vs. standard after contrast reversal vs. standard after changed direction of illumination) ANOVAs for both types of object revealed significant main effects of priming condition (non-handed object: $F(2,36)=4.28, p<0.05$; handed objects: $\mathrm{F}(2,36)=12.44, \mathrm{p}<0.001)$ and rendering condition (non-handed object: $\mathrm{F}(2,36)=6.56, \mathrm{p}<0.01$; handed objects: $\mathrm{F}(2,36)=4.30, \mathrm{p}<0.05)$. There was no significant interaction between the two factors (non-handed object: $\mathrm{F}(4,36)=1.67, \mathrm{p}=0.18$; handed objects: $\mathrm{F}(4,36)=0.34, \mathrm{p}=0.85)$.

To further test for effects of continuous learning, additional one-way ANOVAs were conducted for each of the priming conditions and object types. For the control condition, there was a significant effect of the preceding test involving changed 
rendering on generalization performance in the re-test (non-handed object: $\mathrm{F}(2,12)=12.21, \mathrm{p}<0.001$; handed objects: $\mathrm{F}(2,12)=4.88, \mathrm{p}<0.05)$. As illustrated in Fig. 4a and confirmed by post-hoc Bonferroni comparisons, this was mainly due to the detrimental effect of contrast reversal. Preceding testing with contrast-reversed images significantly impeded generalisation in the re-test for both the handed and non-handed object (all ps <0.05), whereas a change in the direction of illumination left recognition of both types of objects during the re-test unaffected (all ps>0.24).

No significant evidence for continuous learning was found in case of active vision or active touch priming for non-handed object 1 (active vision: $\mathrm{F}(2,12)=0.37$, $\mathrm{p}=0.70$; active touch: $\mathrm{F}(2,12)=1.29, \mathrm{p}=0.31$ ) and handed objects 2 and 3 (active vision: $\mathrm{F}(2,12)=1.42, \mathrm{p}=0.28$; active touch: $\mathrm{F}(2,12)=0.72, \mathrm{p}=0.51)$. For both types of objects generalization performance in the re-test after a preceding test with changed rendering did not differ significantly from that without such test history. This indicates that prior knowledge from active visual or tactual sensation enabled the acquisition of more robust object representations.

\section{DISCUSSION}

We have shown that contrast reversal or a change in direction of illumination may be deleterious to the visual recognition of non-face objects. Yet it is possible to enable invariant recognition by providing observers with prior object knowledge from active sensation (active vision or active touch). Such type of information does not only diminish the effects of changed rendering but allows the construction of object representations that are relatively robust against perturbation by intervening input signals, i.e., continuous learning. 
Our results qualify previous claims of a generic invariance of non-face objects against contrast reversal (Nederhouser et al., 2007). Indeed, the latter would seem to be in stark contrast to what is known from studies in perceptual learning. Discrimination performance typically improves with practice but does not transfer to new stimulus conditions (e.g., Fiorentini \& Berardi, 1980; Karni \& Sagi, 1991; Poggio, Fahle \& Edelman, 1992). Accordingly, perceptual learning in motion discrimination is highly specific to practised directions of motion for difficult discrimination tasks but transfers to new directions when the difficulty is reduced (Liu, 1999). Similarly, Jüttner et al. (2004) have shown that the effects of contrast reversal on visual pattern recognition are determined by task-dependent characteristics of learned representations.

Here we have observed that changes in rendering clearly impair visual object categorisation but prior knowledge from active sensation may facilitate recognition and make it more invariant against changes in rendering. Furthermore, prior knowledge from active sensation may reduce continuous learning. To better understand these results, it is helpful to note that machine learning systems are typically based on the assumption of learning being restricted to a certain time interval (learning phase), whereupon systems are put into operation (working phase). Yet there exist systems that allow learning to take place all the time in an uninterrupted fashion, thus being able to adapt to time-variant environments (Tagscherer and Protzel, 1998; Tagscherer, 2001; Keith-Magee, 2001, unpublished Ph.D. dissertation, Curtin University of Technology, Perth, W.A.). Moreover, there is reason to believe that perceptual categorisation and generalization are adaptive per se (Edelman, 1987). Thus it is not surprising that more reliable information on the physical 3D-structure of the environment from active sensation entails the construction of more invariant and robust internal representations for visual object categorisation. 
The present study differs from earlier work on visual object recognition mainly in two respects. First, a paradigm of category learning involving 2D views from all over the viewing (half-) sphere of non-handed and handed structure-only objects was employed. This precluded recognition from non-accidental object parts by necessitating the construction and maintenance in working memory (see Baddeley, 1986) of object representations conserving at least aspects of 3D-structure. By contrast, Nederhouser and co-workers (2007) used a matching-to-sample paradigm, where participants were presented simultaneously with three object views from same viewpoint of which two portrayed the same object. Thus, observers could rely on image discrimination by comparing contrast invariant shape features. For instance, in Fig. 4a of Nederhouser et al., the distractor and the right matching view show at their lowest central positions the same acute protuberance, which is lacking in the left matching view.

Second, the effects of changed rendering were most conspicuous for handed chiral objects. Concerning this observation, it is helpful to note that mirror-symmetric object 1 can be carried into itself by mirror reflections (3-fold mirror symmetry; see Weyl, 1952). There is evidence that such symmetries facilitate object recognition (Vetter, Poggio \& Bülthoff, 1994). Handed chiral objects 2 and 3 can be carried into themselves only by movements enacted on rigid bodies such as pure rotations (see International Union of Crystallography, 2005), whereas mirror reflections carry them into each other, thus contributing to their confusion. Indeed, physical models of handed chiral objects are disambiguated by spatial transformation in 3D and alignment to a scene-based reference (Hinton \& Parsons, 1988), whereas the recognition of handed objects from 2D projections involves "mental rotation" of internalized models (Shepard \& Metzler, 1971). In brief, symmetry properties facilitate the recognition of non-handed object 1 but impair the recognition of handed chiral objects 2 and 3 . 
The possibility of non-handed object 1 being recognized from about half of its test views by relying on projected symmetries and the three-fold connectivity of one of its sphere parts (Fig. 1b, left) has to be acknowledged. Similarly, about half of the views of handed objects 2 (Fig. 1b, middle) and 3 (Fig. 1b, right) display a "snake-like" structure (introspective reports of participants), thus allowing their distinction against non-handed object 1 but not each other. However, for classifying the remaining "difficult" (introspective reports of participants) views and in particular handedness, observers had to enact the full cycle of image understanding, i.e., the (mental) generation of candidate 3D models from input images (inverse modelling), their rendering back into images (forward modelling), and the corresponding matching process (for formal solutions in the area of machine intelligence see Caelli \& Bischof, 1997; Cheng, Caelli \& Sanchez-Azofeifa, 2006). Given the relative disadvantage of recognising handedness in the absence of prior knowledge and, to some extent, with active vision priming, it can be assumed that observers relied on attributes, or features, in image format under such conditions. The dependence of recognition on rendering conditions would be the consequence of such classification strategies.

We shall then turn to the question of how prior knowledge from active sensation may have affected the construction of object representations. It would have drawn the attention of subjects to 3D structures, thus encouraging them to generate corresponding models (Thoma, Hummel \& Davidoff, 2004). They may have achieved this by encoding temporal sequences of object views (active vision) or exploratory finger and hand movements along the physical object models (active touch). As object palpation directly evokes mental imagery (Critchley, 1953, chap. IV), we can conjecture that some sort of kinetic object traces were stored in multimodal representations (e.g., Zangaladze, Epstein, Grafton \& Sathian, 1999). Subjects may then have inferred 3D structures from 
linking object parts exposed in 2D views to such representations. Related categorisation strategies would have enabled more invariant recognition.

In conclusion, we agree with Nederhouser et al. (2007) in that effects of contrast reversal depend on the nature of internal object representations rather than physical stimulus attributes such as spectral composition (e.g., Hayes, Morrone \& Burr, 1986). The difference in perspective resides in the fact that Nederhouser and co-workers attributed the effect of contrast reversal on recognition to fixed and invariant stimulus categories (face vs. non-face objects), whereas we found it task- or context-dependent. We propose that prior object knowledge from active sensation causes representational shifts as have been reported for face recognition (see Palmeri, Wong, and Gauthier, 2004) and classification of mirror-image patterns (Rentschler \& Jüttner, 2007). Such shifts enable increased invariance of recognition due to an increased use of contrast- or illumination-invariant part-relational object attributes. Moreover, they render object representations more robust, i.e., less sensitive to modification through interfering input signals. 
Acknowledgement: Study supported by Deutsche Forschungsgemeinschaft (DFG) and dedicated to Professor Terrence Michael Caelli on occasion of his retirement from the National Institute of Information Technologies (NICTA) in Canberra, Australia. 


\section{REFERENCES}

Ahmed, N., \& Rao, K.R. (1975). Orthogonal Transforms for Digital Image Processing. Berlin: Springer Verlag.

Albright, T.D. (1995). "My most true mind thus makes mine eye untrue". Trends in Neuroscience 18, 331-333.

Baddeley, A. (1986). Working Memory. Oxford: Oxford University Press.

Biederman, I. (1987). Recognition-by-components: a theory of human image understanding. Psychological Review 94, 115-147.

Biederman, I., \& Cooper, E.E. (1991). Priming contour-deleted images: evidence for intermediate representations in visual object recognition. Cognitive Psychology, 23, 393-419.

Biederman, I., \& Kalocsai, P. (1997). Neurocomputational bases of object and face recognition. Philosophical Transactions of the Royal Society London: Biological Sciences, 352, 1203-1219.

Bülthoff, H.H., \& Edelman, S. (1992). Psychophysical support for a two-dimensional view interpolation theory of object recognition. Proceedings National Academy of Sciences USA, 89, 60-64.

Caelli, T., \& Bischof, W.F. (1997). Machine Learning and Image Interpretation. New York: Plenum Press.

Caelli, T., \& Dreier, A. (1994). Variations on the evidence-based object recognition theme. Pattern Recognition 27, 185-204.

Caelli, T., \& Rentschler, I. (1986). Cross-correlation model for pattern acuity. Journal of the Optical Society of America A, 3, 1948-1956. 
Caelli, T., Hübner, M., \& Rentschler, I. (1986). On the discrimination of micropatterns and textures. Human Neurobiology 5, 129-136.

Caelli, T., Rentschler, I., \& Scheidler, W. (1987). Visual pattern recognition in humans. I. Evidence for adaptive filtering. Biological Cybernetics 57, 233-240.

Cheng, L., Caelli, T., \& Sanchez-Azofeifa, A. (2006). Component optimization for image understanding: A Bayesian approach. IEEE Transactions on Pattern Analysis and Machine Intelligence 28, 684-693.

Cooper, L. A., Schacter, D. L., Ballesteros, S., \& Moore, C. (1992). Priming and recognition of transformed three-dimensional objects: effects of size and reflection. Journal of Experimental Psychology: Learning, Memory, and Cognition, 18, 43-57.

Critchley, M., (1953). The Parietal Lobes. London: Edward Arnold.

Edelman, G.M., (1987) Neural Darwinism. New York: Basic Books.

Fiorentini, A., \& Berardi, N. (1980). Perceptual learning specific for orientation and spatial frequency. Nature 287, 43-44.

Galper, R.E. (1970). Recognition of faces in photographic negative. Psychonomic Science 19, 207-208.

Hayes, A., Morrone, M.C., \& Burr, D.C. (1986). Recognition of positive and negative bandpass-filtered images. Perception 15, 595-602.

Hinton, G. E., \& Parsons, L. M. (1988). Scene-based and viewer-centred representations for comparing shapes. Cognition 30, 1-35.

International Union of Crystallography (2005). Chirality. http://www.iucr.org/iucrtop/comm/cteach/pamphlets/21/node7.html 
Jain, A., \& Hoffman, D. (1988). Evidence-based recognition of objects. IEEE Transactions on Pattern Analysis and Machine Intelligence 10, 783-802.

Jüttner, M., Caelli, T., \& Rentschler, I. (1997). Evidence-based pattern recognition: a structural approach to human perceptual learning and generalization. Journal of Mathematical Psychology 41, 244-258.

Jüttner, M., Langguth, B., \& Rentschler, I. (2004). The impact of context on pattern category learning and representation. Visual Cognition 11, 921-945.

Karni, A. \& Sagi, D. (1991). Where practice makes perfect in texture discrimination: Evidence for primary visual cortex plasticity. Proceedings of the National Academy of Sciences USA 88, 4966-4970.

King, R. B. (2003). Chirality and handedness. The Ruch "shoe-potato" dichotomy in the right-left classification problem. Annals New York Academy of Sciences, 988, 158170.

Liu, Z. (1999). Perceptual learning in motion discrimination that generalizes across motion directions. Proceedings of the National Academy of Sciences USA 96, 1408514087.

Liu, C.H., Collin, C.A., Burton, A.M., \& Chaudhuri, A. (1999). Lighting direction affects recognition of untextured faces in photographic positive and negative. Vision Research 39, 4003-4009.

Nederhouser, M., Yue, X., Mangini, M.C., \& Biederman, I. (2007). The deleterious effect of contrast reversal on recognition is unique to faces, not objects. Vision Research $47,2134-2142$. 
Osman, E., Pearce, A.R., Jüttner, M., \& Rentschler, I. (2000). Reconstructing mental object representations: a machine vision approach to human visual recognition. Spatial Vision, 13, 277-286.

Palmeri, T.J., Wong, A. C.-N., \& Gauthier, I. (2004). Computational approaches to the development of visual expertise. Trends in Cognitive Sciences, 8, 378-386.

Phillips, R.J. (1972). Why are faces hard to recognize in photographic negative? Perception and Psychophysics 12, 425-426.

Poggio, T., Fahle, M., \& Edelman, S. (1992). Fast perceptual learning in visual hyperacuity. Science 256, 1018-1021.

Rentschler, I., \& Jüttner, M. (2007). Mirror-image relations in category learning. Visual Cognition, 15, 211-237.

Rentschler, I., Jüttner, M., \& Caelli, T. (1994). Probabilistic analysis of human supervised learning and classification. Vision Research, 34, 669-687.

Rentschler, I., Gschwind, M., Brettel, H., Osman, E., \& Caelli, T. (2008). Structural and view-specific representations for the categorization of three-dimensional object. Vision Research (in press)

Shepard, R. N., \& Metzler, J. (1971). Mental rotation of three-dimensional objects. Science 171, 701-703.

Subramaniam, S., \& Biederman, I. (1997). Does contrast-reversal affect object recognition? Investigative Ophthalmology and Visual Science 38, 998.

Tagscherer, M. (2001). Dynamische Neuronale Netzarchitektur für Kontinuierliches Lernen. Ph.D. Dissertation, TU Chemnitz, http://archiv.tu-chemnitz.de/pub/2001/0072/ 
Tagscherer, M., \& Protzel P. (1998). Adaptive input-space clustering for continuous learning tasks. Proceedings in Artificial Intelligence - FNS '98, Munich, Germany, pp. $352-358$.

Thoma, V., Hummel, J. E., \& Davidoff, J. (2004). Evidence for holistic representations of ignored images and analytic representations of attended images. Journal of Experimental Psychology 30, 257-267.

Vetter, T., Poggio, T., \& Bülthoff, H.H. (1994). The importance of symmetry and virtual views in three-dimensional object recognition. Current Biology 4, 18-23.

Vuong, Q., Peissig, J., Harrison, M., \& Tarr, M. (2005) The role of surface pigmentation for recognition revealed by contrast reversal in faces and Greebles. Vision Research 45, 1213-1223.

Weyl, H. (1952). Symmetry. Princeton: Princeton University Press.

Zangaladze, A., Epstein, C.M., Grafton, S., \& Sathian, K. (1999). Involvement of visual cortex in tactile discrimination of orientation. Nature 401, 587-590. 


\section{Figure captions:}

Figure 1. 2D views of the set of objects for category learning and generalisation. (a) Achiral (bilaterally symmetric; left) object 1 (O1) and handed chiral (mirror image; centre and right) objects $2(\mathrm{O} 2)$ and $3(\mathrm{O} 3)$. Three spheres formed a rectangular isosceles triangle. The fourth sphere was placed perpendicularly above the centre of one of the base spheres. All spheres were of same size and reflectance. (b) Sample of four 2D views for each of the objects (left). Views obtained from sampling azimuth and polar angle of the viewing (half) sphere in $60^{\circ}$ steps, thus yielding 8 views per object (right). 2 views of object 1 were redundant due to symmetry reasons. Discarding them, 22 views (6 for object 1 and 8 for each of objects 2 and 3) were used for category learning to criterion.

Figure 2. Changed object rendering. (a) For contrast reversal, original intensities were subtracted from the maximum value 255 to obtain reversed intensities. Background intensities remained fixed under these conditions. (b) For a change in the direction of illumination, the default position of the virtual light source was changed from the upper left corner to the lower right corner from the observer's position.

Figure 3. Spatial generalisation under three conditions of rendering and three conditions of priming. Standard rendering (leftmost double bars), contrast reversal (centre), and changed direction of illumination (rightmost double bars). Priming conditions of control (top), active vision (centre), and active touch (bottom). Generalisation performance is given in percent correct classification. Left bar of double bars: Object 1; right bar of double bars: mean value of objects 2 and 3. Error bars: 1 S.E. $(\mathrm{N}=5$; $\mathrm{O} 1)$; mean values of S.E.s $(\mathrm{N}=5$ each; $\mathrm{O} 2$ and $\mathrm{O} 3)$. 
Figure 4. Re-test of spatial generalisation under conditions of standard rendering following spatial generalisation under conditions of contrast reversal (middle double bars), and changed direction of illumination (rightmost double bars). Data for participants, who were initially tested under conditions of standard rendering, reproduced from Fig. 3 (leftmost double bars). Priming conditions of control (top), active vision (centre), and active touch (bottom). Generalisation performance is given in percent correct classification. Left bars of double bars: object 1; right bars of double bars: mean value of objects 2 and 3. Error bars: 1 S.E. $(\mathrm{N}=5$; O1); mean values of S.E.s ( $\mathrm{N}=5$ each; $\mathrm{O} 2$ and $\mathrm{O} 3)$.

Table 1. Duration of category learning to criterion for the three conditions of control, active vision priming, and active touch priming. Mean values over groups of participants and standard errors in number of learning units $(\mathrm{N}=15$, standard rendering; $\mathrm{N}=15$, contrast reversal, and $\mathrm{N}=15$, direction of illumination). 


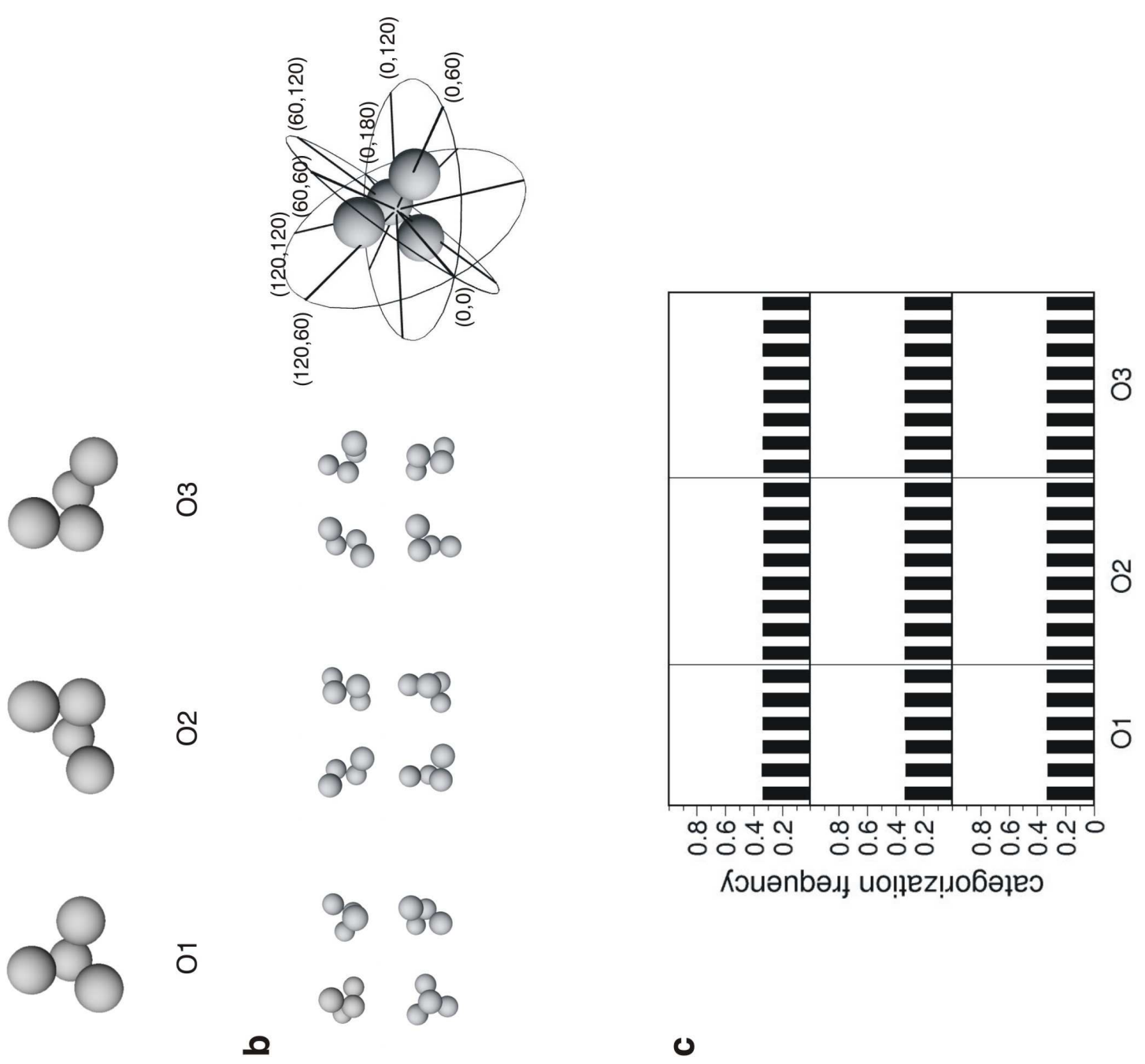

ช

을

0

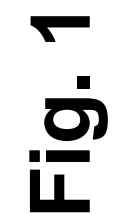



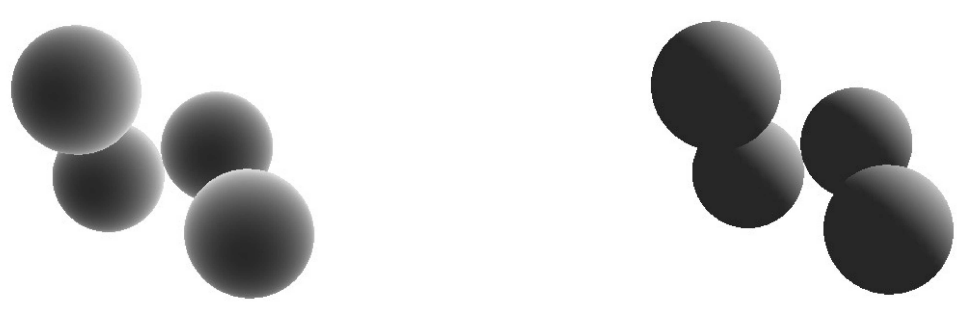

N
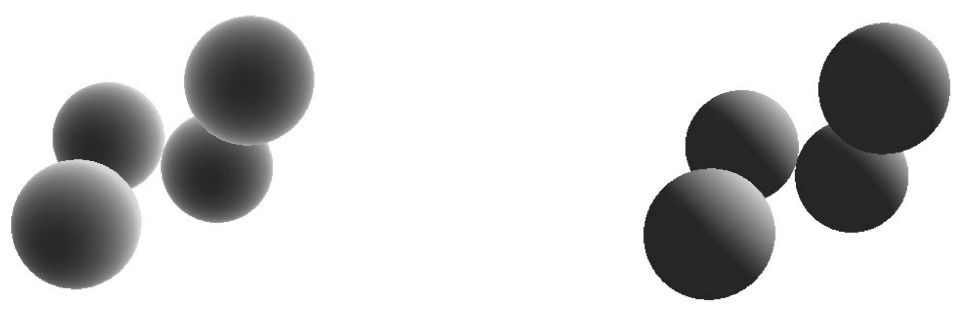

온
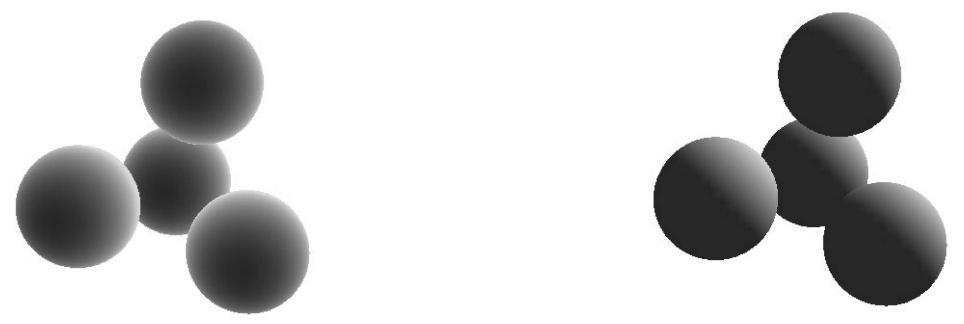

$\sigma$

으 


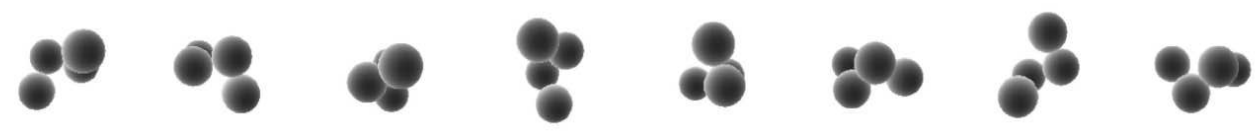

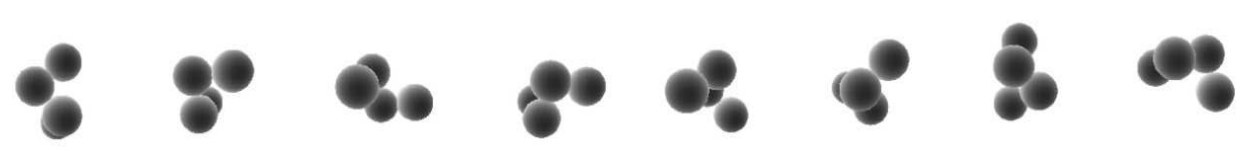

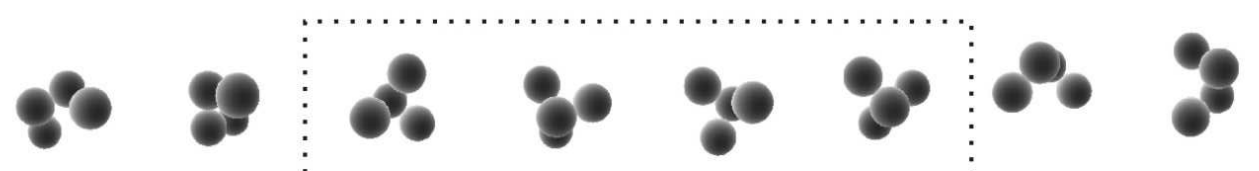

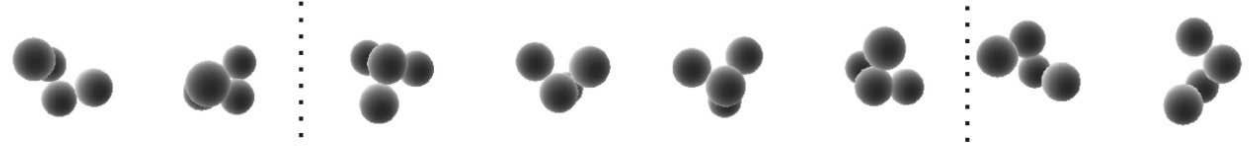

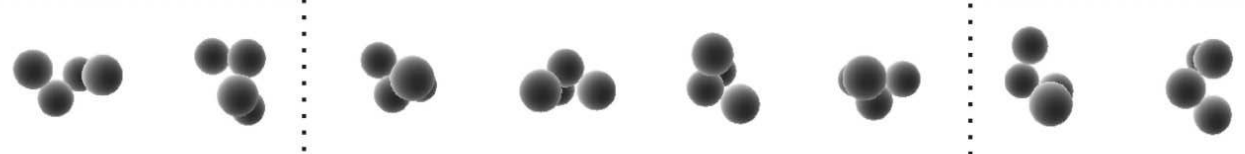

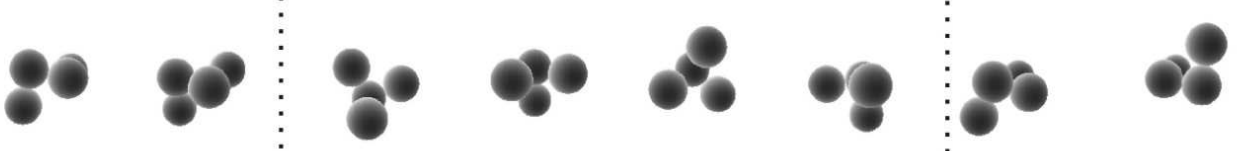

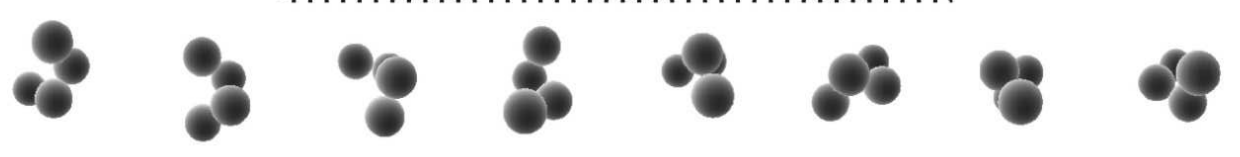

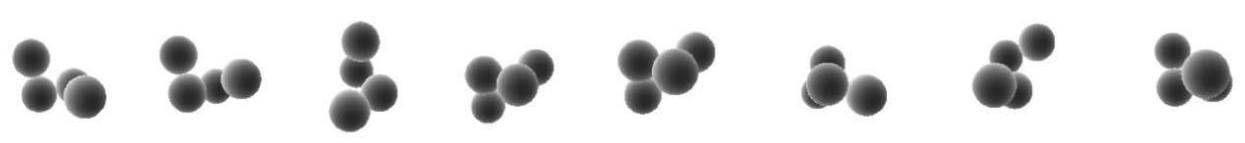

온 


\section{. \\ 운}
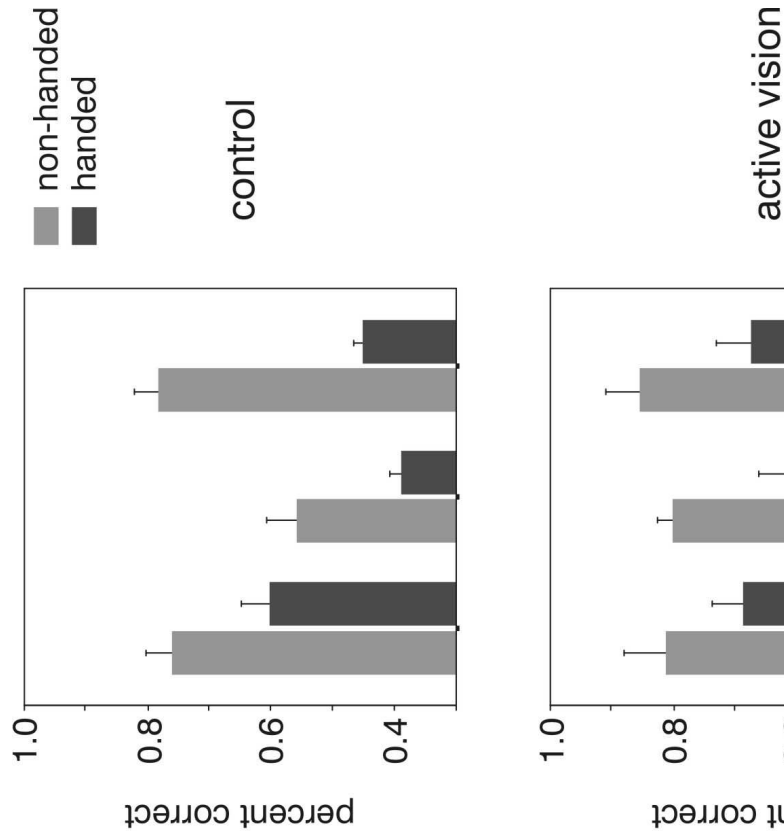

$\sigma$

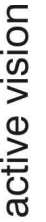

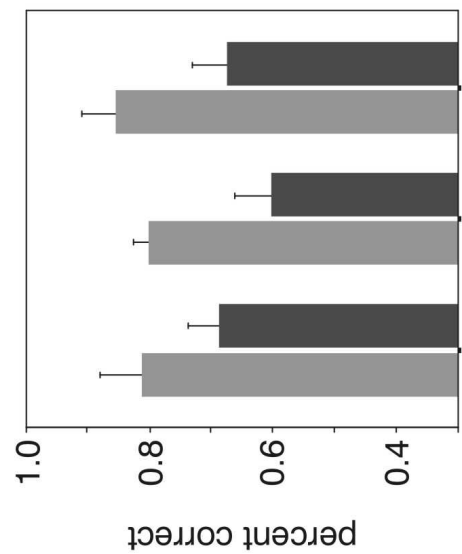

으 $\frac{1}{0}$
0
0
0
0



0 


\section{8 \\ ㅇํำ}

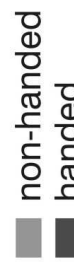

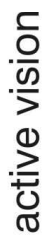

$\frac{1}{0}$
0
0
0
0
0
0

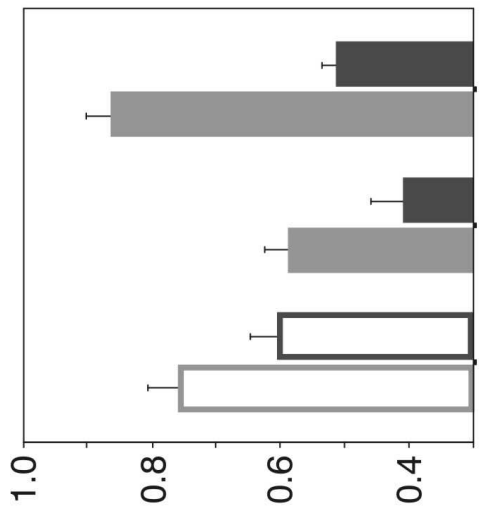

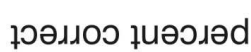

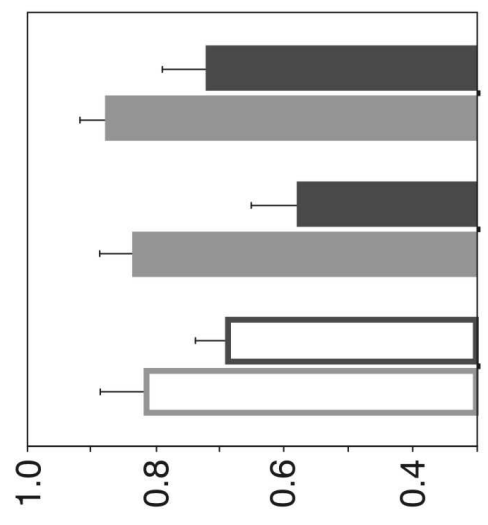

นวәมоว ұนәวләd

2

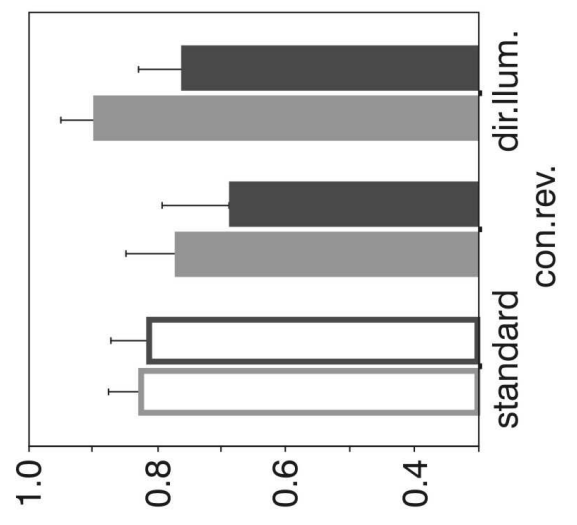

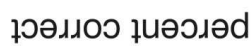

( 


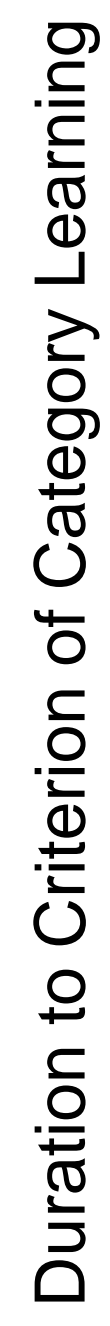
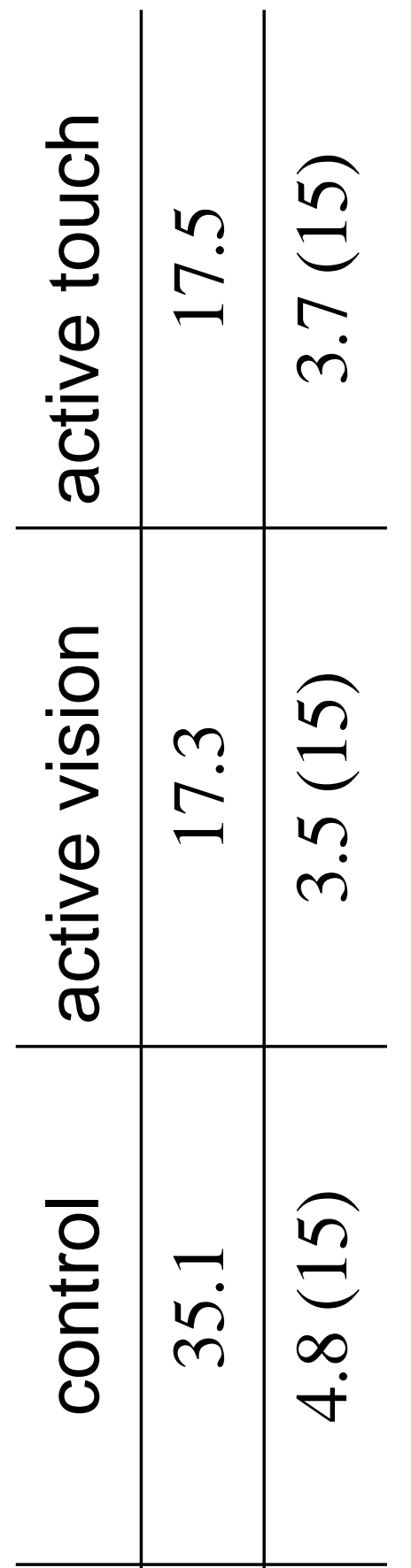

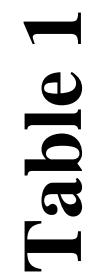

든

Е 